\title{
Nondestructive quantification of chemical and physical properties of fruits by time-resolved reflectance spectroscopy in the wavelength range 650-1000 nm
}

\author{
Rinaldo Cubeddu, Cosimo D'Andrea, Antonio Pifferi, Paola Taroni, \\ Alessandro Torricelli, Gianluca Valentini, Colin Dover, David Johnson, \\ Margarita Ruiz-Altisent, and Constantino Valero
}

\begin{abstract}
Time-resolved reflectance spectroscopy can be used to assess nondestructively the bulk (rather than the superficial) optical properties of highly diffusive media. A fully automated system for time-resolved reflectance spectroscopy was used to evaluate the absorption and the transport scattering spectra of fruits in the red and the near-infrared regions. In particular, data were collected in the range $650-1000 \mathrm{~nm}$ from three varieties of apples and from peaches, kiwifruits, and tomatoes. The absorption spectra were usually dominated by the water peak near $970 \mathrm{~nm}$, whereas chlorophyll was detected at $675 \mathrm{~nm}$. For all species the scattering decreased progressively with increasing wavelength. A best fit to water and chlorophyll absorption line shapes and to Mie theory permitted the estimation of water and chlorophyll content and the average size of scattering centers in the bulk of intact fruits. $\bigcirc 2001$ Optical Society of America

OCIS codes: $170.5280,170.7050,300.1030,290.0290,300.6500$.
\end{abstract}

\section{Introduction}

The nondestructive assessment of the quality of fruits and vegetables is increasing in importance for both production and marketing. At present, destructive chemical-physical and mechanical methods are routinely used to obtain information on internal structure and properties, whereas noninvasive techniques for quality detection often rely only on exter-

R. Cubeddu (rinaldo.cubeddu@polimi.it), C. D'Andrea, A. Pifferi, P. Taroni, A. Torricelli, and G. Valentini are with the Istituto Nazionale per la Fisica della Materia, Dipartimento di Fisica and Centro di Elettronica Quantistica e Strumentazione Elettronica. Consiglio Nazionale delle Ricerche, Politecnico di Milano, Piazza Leonardo da Vinci 32, I-20133 Milan, Italy. C. Dover and D. Johnson are with Horticulture Research International, East Malling, West Malling, Kent. ME19 6BJ, UK. M. Ruiz-Altisent and $C$. Valero are with the Departamento de Ingenieria Rural, Escuela Técnica Superior de Ingenieros Agrónomos, Universidad Politécnica de Madrid, Av/Complutenses/n. 28040 Madrid, Spain

Received 13 June 2000 ; revised manuscript received 29 September 2000 .

$0003-6935 / 01 / 040538-06 \$ 15.00 / 0$

(1) 2001 Optical Society of America nal appearance ${ }^{1,2}$ (e.g., size, shape, color, and external defects).

A promising exception is continuous-wave (cw) near-infrared (NIR) reflectance spectroscopy. 3 The NIR spectra are obtained nondestructively and depend not only on the presence of particular substances but also on their concentration, thus providing useful data for both identification and quantification. A good correlation with NIR reflectance was observed for the sugar content in apples 4 and for firmness in kiwifruits. 5 However, both absorption and scattering influence the $\mathrm{cw}$ reflectance signal, and their effects cannot be easily separated with a single measurement.

Time-resolved reflectance spectroscopy (TRS) is a novel nondestructive means for the complete optical characterization of highly diffusive media, i.e., for the evaluation of the absorption coefficient $\mu_{\alpha}$ and the transport scattering coefficient $\mu_{s}^{\prime}$. In particular, TRS is gaining acceptance in biomedicine for the noninvasive investigation of biological tissues. ${ }^{6-9}$ Similarly, it could provide useful insight in to the internal properties of most fruits.

In TRS a short light pulse is injected into the medium to be analyzed. Because of the absorption and 
the scattering events occurring for the photons during their propagation, the diffusely reflected pulse is attenuated, broadened, and delayed. Consequently the best fit of its time distribution with a theoretical model of light propagation allows the simultaneous evaluation of both the optical coefficients $\mu_{\alpha}$ and $\mu_{s}$ '. Light penetration into most fruits and vegetables can be as great as $1 \mathrm{~cm}$, depending on the optical properties. ${ }^{10}$ Consequently the measurement probes bulk rather than superficial properties and could provide useful information on internal quality.

In the present study, for the first time to our knowledge, a fully automated system for TRS was applied to the optical characterization of several species and varieties of fruits in the red and the NIR ranges $(650-1000 \mathrm{~nm})$. Best fits of the absorption spectrum and the transport scattering spectrum to the literature spectra and to approximate Mie theory were performed to quantify tissue constituents and structure, respectively.

\section{Materials and Methods}

\section{A. System Setup}

A synchronously pumped mode-locked dye [4dicyanomethylene-2-methyl-6-( $p$-dimethylaminostyryl)-4H-pyran] laser (CR-599, Coherent, Calif) was used as the excitation source from 650 to $695 \mathrm{~nm}$ with a repetition rate of $76 \mathrm{MHz}$, whereas an actively mode-locked titanium:sapphire laser (3900, SpectraPhysics, Calif.) provided light in the wavelength range of 700 to $1000 \mathrm{~nm}$ with a repetition rate of 100 MHz.

Two 1-mm plastic-glass fibers (PCS1000W, Quartz et Silice, France) delivered light into the sample and collected the reflected photons. For the measurements reported here, the power density at the distal end of the illumination fiber was always limited to less than $10 \mathrm{~mW}$. A home-built-holder allowed the fibers to be positioned $1.5 \mathrm{~cm}$ apart, parallel to each other, normal to and in contact with the sample surface.

A double microchannel plate photomultiplier (R1564U with S1 photochatode, Hamamatsu, Japan) and an electronic chain for time-correlated singlephoton counting (TCSPC) were used for detection. A small fraction of the incident beam was coupled to a 1-mm fiber (PCS1000W, Quartz et Silice, France) and fed directly to the photomultiplier to correct for any time drift of the instrumentation.

Overall, the system transfer function was $<120$ and $<180$ ps (FWHM) in the red and the NIR, respectively.

The instrumentation was fully automated, and the analysis and display of the measured spectra were performed in real time. A personal computer controlled the laser tuning and power, the monochromator scanning, and the optimization of the system transfer function by adjusting the laser cavity length. The overall measurement time (for data acquisition and system adjustment) was $8-10 \mathrm{~s} /$ wavelength.

\section{B. Data Analysis}

We constructed the transport scattering and absorption spectra by plotting, as a function of wavelength, the values of $\mu_{s}^{\prime}$ and $\mu_{\alpha}$ obtained from fitting the experimental data with a standard solution of the diffusion approximation to the radiative transport equation for a semi-infinite homogeneous medium. The extrapolated boundary condition was used, 11 and the diffusion coefficient $D$ was taken to be independent of the absorption properties of the medium (i.e., $\left.D=1 / \mu_{s}\right)$, in agreement with Furutsu and Yamada. ${ }^{12}$ The theoretical curve was convoluted with the system transfer function and normalized to the area of the experimental curve. The fitting range included all points with a number of counts higher than $10 \%$ of the peak value on the rising edge of the curve and $1 \%$ on the tail. The best fit was reached with a Levenberg-Marquardt algorithm ${ }^{13}$ when both $\mu_{s}^{\prime}$ and $\mu_{a}$ were varied to minimize the reduced $x^{2}$.

With the setup and the theoretical model used, the accuracy in the absolute estimate of both $\mu_{s}^{\prime}$ and $\mu_{\alpha}$ is usually better than $10 \%$. However, in the range of measured values of the optical coefficients, the error in the assessment of the absorption line shape is considerably smaller $(<2 \%) .^{14.15}$

To quantify the percentage volume of water and the chlorophyll a content in the bulk of the intact fruits, the absorption spectrum was best fitted with a linear combination of the line shapes of water ${ }^{16}$ and of chlorophyll $a^{1 \%}$.

$$
\mu_{u}(\lambda)=\sum_{i} c_{i} \varepsilon_{i}(\lambda)
$$

where $c_{i}$ is the concentration (free parameter in the fitting procedure) and $\varepsilon_{i}(\lambda)$ is the specific absorption of the $i$ th constituent. Potential contributions from other minor tissue absorbers were all combined and taken into account by an addition to the linear combination of a wavelength-independent spectrum of free amplitude $k$. The best fit was reached with a generalized reduced gradient algorithm ${ }^{18}$ when both $c_{i}$ and $k$ were varied to minimize the reduced $\chi^{2}$.

Further information could be obtained from the transport scattering spectra. For a homogeneous sphere of radius $r$, Mie theory predicts the wavelength dependence of the scattering and the relation between scattering and sphere size. Under the hypothesis that the scattering centers are homogeneous spheres behaving individually, the relationship between $\mu_{s}^{\prime}$ and wavelength $\lambda$ can be empirically described ${ }^{19}$ as

$$
\mu_{s}^{\prime}(\lambda)=a x^{b}
$$

where the size parameter $x$ is defined as $x=$ $2 \pi m n_{m} \lambda^{-1}$, the refraction index of the medium $n_{m}$ is chosen to be 1.35 , and $a$ and $b$ are free parameters. In particular, $\alpha$ is proportional to the density of the scattering centers, and $b$ depends on their size. Moreover, $b$ can be empirically expressed ${ }^{20}$ as a third- 


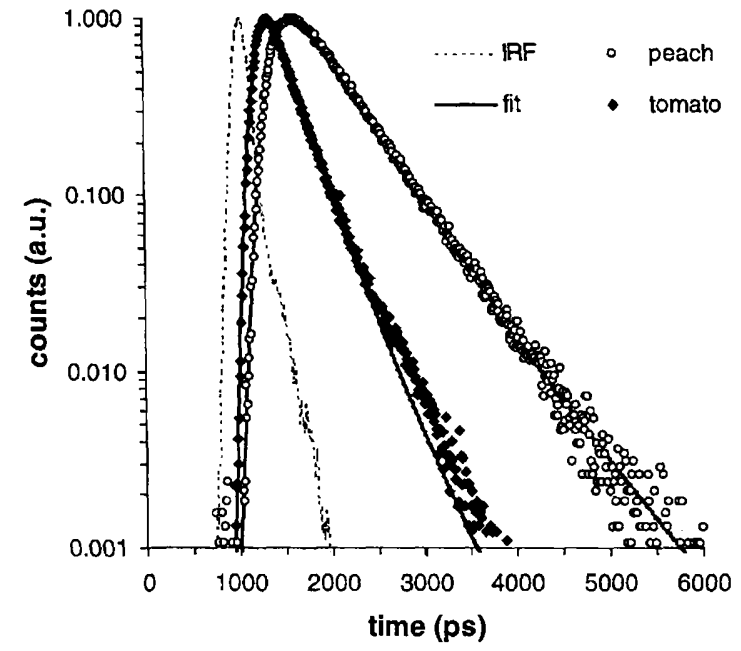

Fig. 1. Typical TRS curves for a peach (open circles) and for a tomato (filled diamonds), instrumental response function (IRF, dashed curve), and best fit to the theoretical model (solid curve).

order polynomial function of $r$; therefore the estimate of $b$ can yield the sphere radius $r$.

\section{Experimental Procedure}

Time-resolved reflectance measurements were carried out every $5 \mathrm{~nm}$ from 650 to $1000 \mathrm{~nm}$ on apples (Golden Delicious, Granny Smith, and Starking Delicious), peaches (yellow flesh), kiwifruits (Hayward), and tomatoes (Daniela). For each species and variety, at least five items were tested. On each sample, in general, measurements were performed in two positions, corresponding to the most and the least colored side.

\section{Results and Discussion}

Figure 1 shows typical TRS curves for a peach and a tomato, the corresponding best fit with the theoretical model, and the instrumental response function. Because of the absorption and scattering events occurring to the photons during their propagation, the diffusely reflected pulse (i.e., the TRS curve) is attenuated, broadened, and delayed with respect to the injection time. In the case of the peach, because the pulp of the fruit extends for at least $2-3 \mathrm{~cm}$, photons do not penetrate into the core but are totally confined in the pulp. Therefore the peach is, to a first approximation, a homogeneous turbid medium. This assumption is confirmed by the good agreement between the theoretical model and the experimental data. In the case of the tomato we again observe that the broadening of the remitted pulse, although it is less marked than in the previous case, confirms that photon propagation into tissue is a diffusional process. However, the tomato consists of a layer with more dense properties followed by the common cavities partially filled by a more clear substance containing the seeds. Because the thickness of the dense layer is less than $1 \mathrm{~cm}$, photons also propagate in the clear region. The abrupt change in the optical

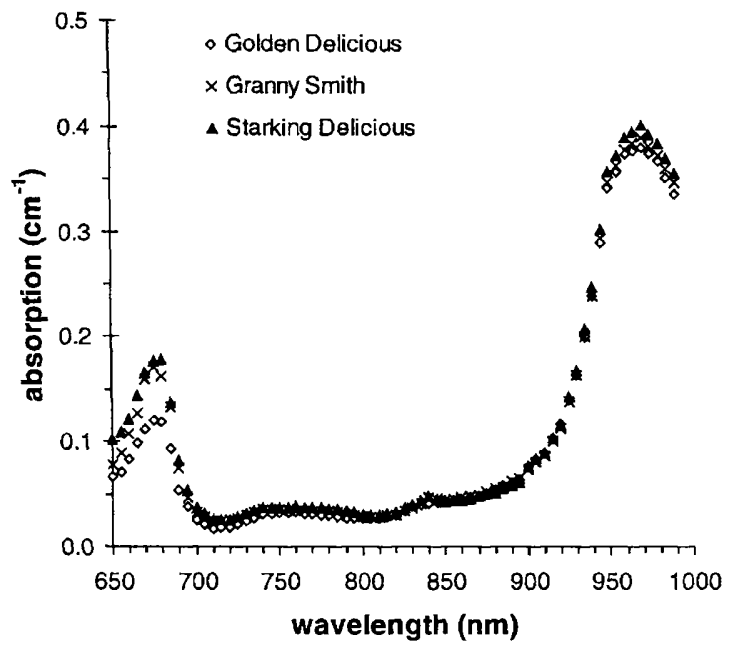

Fig. 2. Typical absorption spectra of Golden Delicious apple (diamonds), Granny Smith apple (crosses), and Starking Delicious apple (triangles).

properties from one region to the other could be revealed by the anomalous behavior of late photons. As a consequence, the fitted $\mu_{c}$ and $\mu_{s}{ }^{\prime}$ in this case should be considered average values over the two regions.

Typical absorption spectra of different varieties of apples (Golden Delicious, Granny Smith, and Starking Delicious) are shown in Fig. 2. Qualitatively similar results were obtained for the three varieties of apples. The absorption spectrum was dominated by the water peak, centered near $970 \mathrm{~nm}$, with an absolute value of $-0.4 \mathrm{~cm}^{-1}$. Minor absorption features of water were usually detected near 740 and $835 \mathrm{~nm}$, where the absorption coefficient was low $\left(0.05 \mathrm{~cm}^{-1}\right)$. A significant absorption peak $(0.12-$ $0.18 \mathrm{~cm}^{-1}$ ) was found at $675 \mathrm{~nm}$, corresponding to chlorophyll $a$.

Figure 3 shows the absorption spectra of yellow peach, tomato, and kiwifruit. Both the line shape and the absolute value of the absorption spectra of peach and tomato are quite similar to those of the apples shown in Fig. 2. However, for kiwifruit, as expected from the visual appearance of its flesh, chlorophyll $a$ absorption was considerable, with a maximum value up to $\sim 10$ times the maximum in other fruits (data not shown).

Information on the water and chlorophyll content can be obtained from absorption spectra fitted to the line shape of these pure constituents. Figure 4 shows a typical example of a fit for the absorption spectrum of a Starking Delicious apple to the line shapes of water and chlorophyll $a$. Table 1 reports the chlorophyll $a$ and water content in different fruits. In all cases a $0.02-0.03-\mathrm{cm}^{-1}$ contribution was added by the flat background spectrum. To a first approximation this contribution could be attributed to the absorption of carotenoids and anthocyanines. These chromophores, in fact, show absorption bands at wavelengths shorter than 500 


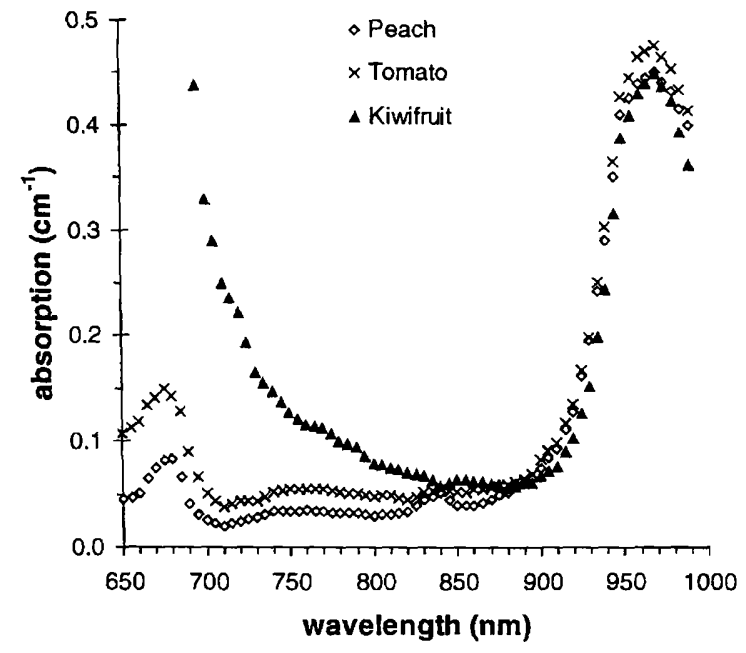

Fig. 3. Typical absorption spectra of peach (diamonds), tomato (crosses), and kiwifruit (triangles).

and $600 \mathrm{~nm}$, respectively. ${ }^{21}$ In the wavelength range under investigation $(650-1000 \mathrm{~nm})$ their contribution is definitely smaller. Thus it would not be feasible to recover their concentrations from our data. The assumption of a flat background spectrum is a first-order approximation of their contribution. In

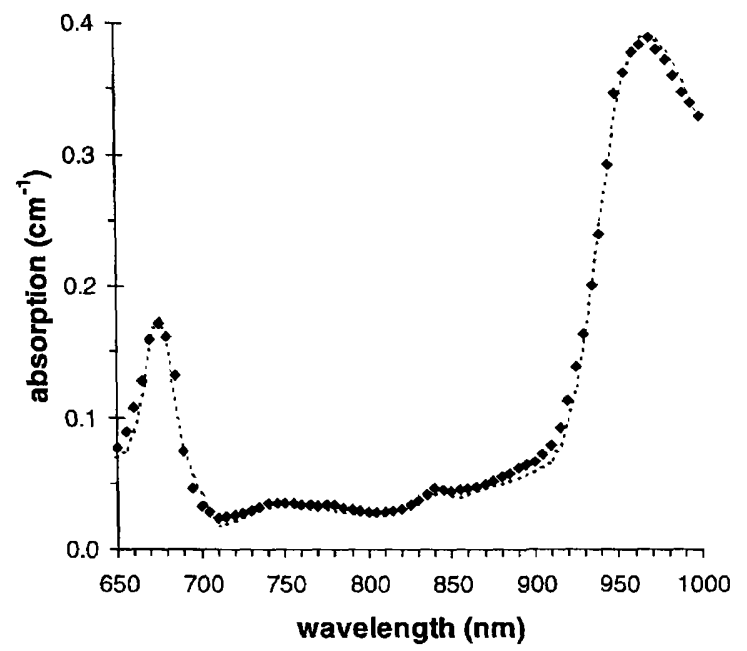

Fig. 4. Best fit of the absorption spectrum of a Starking Delicious apple to the line shape of water ${ }^{16}$ and chlorophyll $a .{ }^{17}$

Table 1. Chlorophyll a and Water Content in Different Fruits as Derived from the Best Fit of the Absorption Spectrum to the Line Shape of the Constituents

\begin{tabular}{lcc}
\hline \multicolumn{1}{c}{ Fruit } & Chlorophyll $a(\mu \mathrm{M})$ & Water $(\%)$ \\
\hline Golden Delicious apple & 0.70 & 81.5 \\
Granny Smith apple & 1.32 & 80.9 \\
Starking Delicious apple & 0.96 & 82.6 \\
Peach & 0.49 & 93.8 \\
Tomato & 0.52 & 95.0 \\
Kiwifruit & 6.91 & 98.8 \\
\hline
\end{tabular}

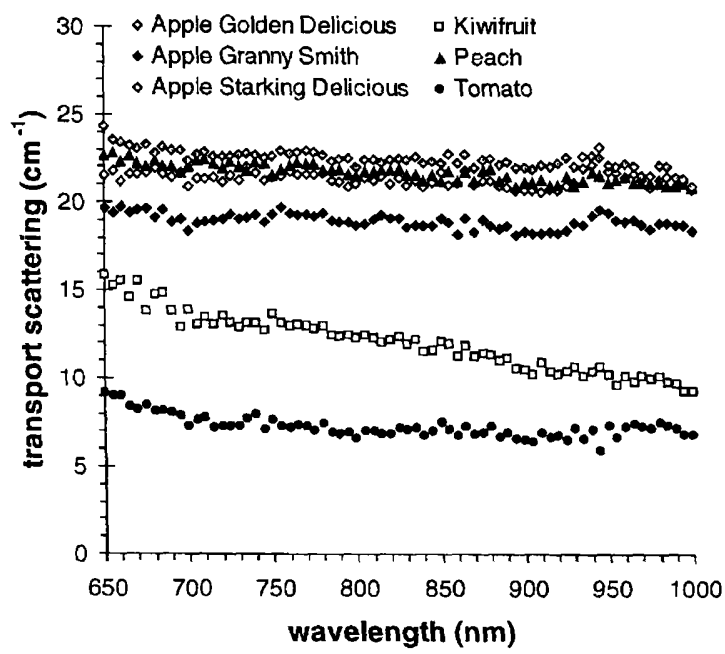

Fig. 5. Typical transport scattering spectra of Starking Delicious (gray diamonds), Golden Delicious (open diamonds), Granny Smith (filled diamonds), peach (filled triangles), tomato (filled circles), and kiwifruit (open squares).

agreement with the different water/fiber ratios in distinct species, a higher $(>90 \%)$ water content was detected in kiwifruits, tomatoes, and peaches than in apples $(-80 \%)$. On the other hand, the chlorophyll content in the pulp of fruits varies from $\sim 0.5 \mu \mathrm{M}$ in peaches and tomatoes to $-1.0 \mu \mathrm{M}$ in apples, and it rises $\sim 7.0 \mu \mathrm{M}$ in kiwifruits. Preliminary data obtained from apples suggest that the chlorophyll content could be a useful parameter for testing the ripening stage. A series of measurements performed over several days on the same fruits showed a progressive decrease in red absorption, in agreement with the gradual reduction in the chlorophyll content with postharvest ripening. ${ }^{2 \%}$

The scattering properties for all the species considered showed no particular spectral features. Typical examples are shown in Fig. 5. The value of the transport scattering coefficient decreased progressively with increasing wavelength, with different slopes depending on the fruit. Apples and the peach had the highest $\mu_{s}^{\prime}\left(-20-24 \mathrm{~cm}^{-1}\right.$ at $\left.700 \mathrm{~nm}\right)$, whereas $\mu_{s}^{\prime}$ was lower for the kiwifruit $\left(-13 \mathrm{~cm}^{-1}\right.$ at $700 \mathrm{~nm})$ and the tomato $\left(-7 \mathrm{~cm}^{-1}\right.$ at $\left.700 \mathrm{~nm}\right)$. The transport scattering spectrum of the kiwifruit was noisier than the spectrum of the apples, particularly in the 650-750-nm region, where the high absorption of chlorophyll reduced the accuracy of the evaluation of transport scattering by TRS measurements. ${ }^{15}$

Figure 6 shows the best fit to Mie theory for the transport scattering spectra of a Granny Smith apple, a peach, and a kiwifruit. The $\log -\log$ plot was used to make evident the power relationship between transport scattering and wavelength in Eq. (2). The estimated average sizes $r$ of scattering centers in different fruits are shown in Table 2. It was observed that $a$ and $b$ varied in the range $3-18 \mathrm{~cm}^{-1}$ and $0.1-1$, respectively. This suggests that different fruits have different densities and average dimensions of 


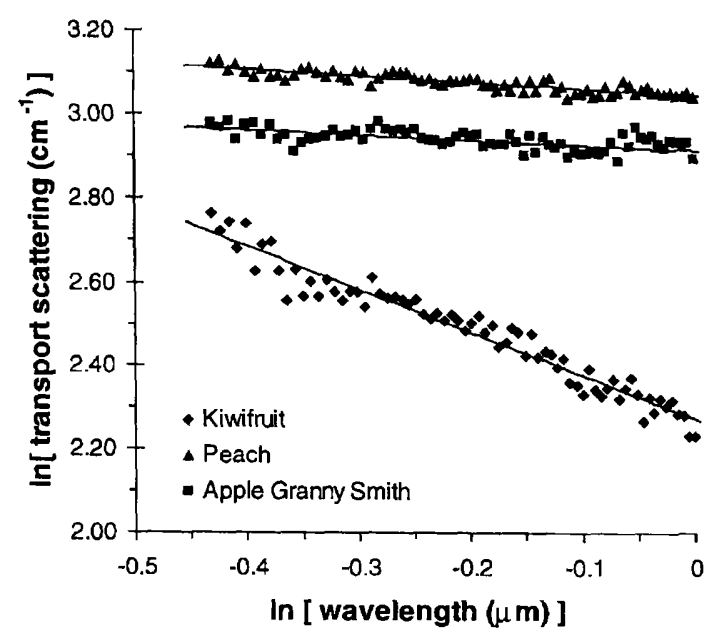

Fig. 6. Best fit of the transport scattering spectrum to the approximation of Mie theory [Eq. (2)]: Granny Smith apple (squares), peach (triangles), and kiwifruit (diamonds).

scattering centers (the range for $r$ is $0.27-0.76 \mu \mathrm{m}$ ). It is worth noting that these parameters do not assess the real size of scattering centers in the tissue; rather, they are average equivalent parameters. Still, they are likely related to physical or chemical fruit characteristics such as firmness or sugar content.

In general, the absorption spectrum of a sample is clearly determined by the nature and the concentration of its constituents, whereas the scattering properties are expected to be related to its structure. This suggests that optical techniques could have a wide diagnostic potential in the assessment of internal structure and quality: The absorption coefficient at certain wavelengths could provide information on the content of sugar, water, chlorophyll, etc., and the scattering coefficient could be used to estimate firmness.

TRS is a nondestructive optical technique that uses only low-intensity light. The number of photons surviving propagation into a turbid medium as well as their trajectories depend on the optical properties (i.e., absorption and transport scattering coefficients) of the traversed medium. Consequently, the estimates of the optical coefficients obtained by TRS are average values over the volume crossed by photon

Table 2. Parameters $a$ and $b$ as Obtained from the Best Fit of the Transport Scattering Spectrum to $\mu_{s}^{\prime}=a x^{b}$ and Equivalent Average Radius $r$ of Scattering Centers ${ }^{a}$

\begin{tabular}{lccc}
\hline \multicolumn{1}{c}{ Fruit } & $a\left(\mathrm{~cm}^{-1}\right)$ & $b$ & $r(\mu \mathrm{m})$ \\
\hline Starking Delicious apple & 17.4 & 0.12 & 0.759 \\
Golden Delicious apple & 17.2 & 0.10 & 0.764 \\
Granny Smith apple & 14.3 & 0.14 & 0.757 \\
Peach & 14.4 & 0.20 & 0.740 \\
Kiwifruit & 4.5 & 0.95 & 0.266 \\
Tomato & 2.9 & 0.48 & 0.591 \\
\hline
\end{tabular}

a The size parameter $x$ is defined as $x=2 \pi r n_{m} \lambda^{-1}$, with the refractive index of the medium $n_{m}$ chosen to be $1.35 .{ }^{19}$ trajectories and refer to the bulk of the medium and not to the surface layers. In the fruit species and wavelength range considered in this study, the $a b-$ sorption coefficient did not usually exceed $1 \mathrm{~cm}^{-1}$ and was often much lower (see below), and the transport scattering coefficient was typically lower than 25 $\mathrm{cm}^{-1}$. This implies a probed volume depth of the order of $0.5-1.0 \mathrm{~cm}$ with $1.5-\mathrm{cm}$ spacing between injection and collection fibers. Thus, at least for thinskinned fruits, the skin should not significantly influence the assessment of the internal optical properties with TRS. This was shown previously by measurements on apples with red (Starking Delicious), yellow (Golden Delicious), and green (Granny Smith) skins. For all varieties, comparable values of absorption and scattering were obtained from the same samples before and after peeling. ${ }^{22}$

This preliminary study was performed in a complex laboratory facility for time-resolved measurements in the wavelength range $650-1000 \mathrm{~nm}$, based on the TCSPC technique. A commercial device for quantification of fruits' physical and chemical parameters could result from a dramatic scaling down of this instrumentation. Pulsed diode lasers and compact boards for TCSPC are available on the market at reasonable costs. Because chlorophyll $a$ and water seem to be the main compounds contributing to absorption in the wavelength range considered here, a two-wavelength device should be able to estimate their absolute concentrations from the appropriate absorption coefficient, and at the same time it would be able to derive information on tissue internal structure from the interpretation of the transport scattering coefficient with approximation to Mie theory.

\section{Conclusion}

We have shown that TRS can be used for the nondestructive assessment of the internal optical properties of intact fruits. For all the fruits considered, the absorption line shapes were determined by the spectral features of chlorophyll $a$ and water, with peaks at 675 and $970 \mathrm{~nm}$, respectively. The transport scattering coefficient decreased progressively with increasing wavelength. The quantification of the water and the chlorophyll content and the estimation of the average size of the scattering centers were obtained by a best fit of the absorption spectrum and the transport scattering spectrum to the literature spectra and to approximate Mie theory, respectively.

Research is presently in progress to provide insight into the correlation between optical properties and physical-chemical and mechanical parameters involved in the definition of fruit quality.

The research was partially supported by the European Community Commission Service DGXII (contract FAIR CT96-1060).

\section{References}

1. P. Chen, "Quality evaluation tecknology for agricultural products," in Praceedings of the International Conference on Agri- 
cultural Machinery Engineering (Korean Society for Agriculture and Machinery, Seoul, 1996), pp. 171-204.

2. J. A. Abbott, "Quality measurement of fruits and vegetables," Post-harvest Biol. Technol. 15, 207-225 (1999).

3. S. Gunasekaran, M. R. Paulsen, and G. C. Shove, "Optical methods for non-destructive quality evaluation of agricul. tural and biological materials," J. Agric. Eng. Res. 32, 209-241 (1985).

4. J. Lammertyn, B. Nicolai, K. Ooms. V. De Smedt, and J. De Baerdemaeker, "Non-destructive measurement of acidity, soluble solid, and firmness of jonagold apples using NIRspectroscopy," Trans. Am. Soc. Agric. Eng. 41, 1089-1094 (1998).

5. V. A. McGlone, H. Abe, and S. Kawano, "Kiwifruit firmness by near infrared light scattering," J. Near Infrared Spectrose. 5, $83-89(1997)$

6. M. S. Patterson, B. Chance, and B. C. Wilson, "Time resolved reflectance and transmittance for the non-invasive measurement of tissue optical properties," Appl. Opt. 28, 2331-2336 (1989).

7. S. L. Jacques, "Time-resolved reflectance spectroscopy in turbid tissues," IEEE Trans. Biomed. Kng. 36, 1155-1161 (1989).

8. B. C. Wilson, and S. L. Jacques, "Optical reffectance and transmittance of tissues: principles and applications," IEEE $J$. Quantum Electron. 26, 2186-2199 (1990).

9. A. Yodh, and B. Chance, "Spectroscopy and inaging with diffusing light," Phys. Today (March, 1995), pp. 34-40.

10. S. Feng. F. Zeng, and B. Chance, "Photon migration in the presence of a single defect: a perturbation analysis," Appl. Opt. 34, 3826-3837 (1995).

11. R. C. Haskell, L. O. Svasaand, T.-T. Tsay, T.-C. Feng, M. S. McAdams, and B. J. Tromberg, "Boundary conditions for the diffusion equation in radiative transfer," J. Opt. Soc. Am. A 11, $2727-2741$ (1994).

12. K. Furutsu and Y. Yamada, "Diffusion approximation for a dissipative random medium and the applications," Phys. Rev. E 50,3634-3640 (1994).
13. W. H. Press, S. A. Teukolsky, W. T. Vetterling, and B. P. Flannery, Numerical Recipes in $C$ : The Art of Scientific Computing (Cambridge U. Press, New York, 1992).

14. R. Cabeddu, M. Musolino, A. Pifferi, P. Taroni, and G. Valentiri, "Time-resolved reflectance: a systematic study for application to the optical characterization of tissues," IEEE IJ. Quantum Electron. 30, 2421-2430 (1994).

15. R. Cubeddu, A. Pifferi, P. Taroni, A. Torricelli, and G. Valentini, "Experimental test of theoretical models for time-resolved reflectance," Med. Phys. 23, 1625-1683 (1996).

16. G. M. Hale and M. R. Querry, "Optical constants of water in the $200 \mathrm{~nm}$ to $200 \mathrm{~mm}$ wavelength region," Appl. Opt. 12, $555-563(1973)$

17. L. L. Shipman, T. M. Cotton, J. R. Norris, and J. J. Katz, "An analysis of the visible absorption spectrum of chlorophyll $a$ monomer, dimer and oligomer in solution," J. Am. Chem. Soc, 98, 8222-8230 (1979).

18. L. S. Lasdon, A. D. Waren, A. Jain, and M. Ratner, "Design and testing of a generalized reduced gradient code for nonlinear programming," ACM (Assoc. Comput. Mach.) Trans. Math. Software 4, 34-50 (1978).

19. J. R. Mourant, T. Fuselier, I. Boyer, T. M. Johnson, and I. J. Bigio, "Predictions and measurements of scattering and absorption over broad wavelength ranges in tissue phantoms," Appl. Opt. 36, 949-95? (1997).

20. M. K. Nilsson, C. Sturesson, D. L. Liu, and S. AnderssonEngels, "Changes in spectral shape of tissue optical properties in conjunction with laser-induced thermotherapy," Appl. Opt. $37,1256-1267$ (1998).

21. J. Gross, Pigments in Fruits (Acadenic, London, 1987), Chaps. 2 and 3.

22. R. Cubeddu, A. Pifferi, P. Taroni, A. Torricelli, G. Valentini, M. Ruiz-Altisent, C. Valero, and C. Ortiz, "Non-destruetive measurements of the optical properties of fruits by means of time-resolved reflectance," in Optical Tomography and Spectroscopy of Tissue MI, B. Chance, R. R. Alfano, and B. J. Tromberg, eds., Proc. SPIE 3597, 445- 449 (1999). 